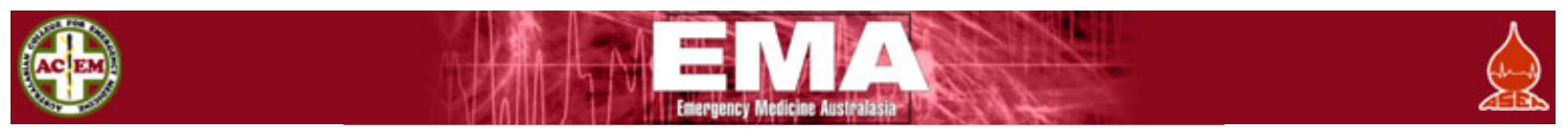

\title{
Emergency department access block occupancy predicts delay to surgery in patients with fractured neck of femur
}

\author{
Drew Richardson ${ }^{1,2}$ and Kristin LH McMahon ${ }^{1}$ \\ ${ }^{1}$ Medical School, Australian National University, ${ }^{2}$ Emergency department, The Canberra Hospital, \\ Garran, Australian Capital Territory, Australia
}

\section{Abstract}

Objective:

The present study aimed to identify any relationship between existing access block occupancy $(\mathrm{ABO})$ at the time of patient presentation and delay to definitive procedure.

Methods:

Retrospective descriptive cohort study of all patients aged over 50 years with an ED diagnosis of fractured neck of femur admitted through a tertiary ED over 2 years. The independent variable was the $\mathrm{ABO}$ at the start of the hour in which the patient presented, derived from existing ED records, and expressed as the quartile for that hour of the day. The dependent variable was start of surgery more than $24 \mathrm{~h}$ after arrival without a documented reason for delay. The data abstractor was blinded to the ABO.

Results:

All 442 diagnoses of fractured neck of femur recorded in the ED were reviewed, 73 were excluded (16 age, 5 misdiagnosis, 31 no surgery, 21 documented medical reasons for delay). There was a significant relationship between $\mathrm{ABO}$ quartile and the rate of delay to surgery ranging from $54 \%(95 \% \mathrm{CI} 43-63 \%)$ for those presenting in the lowest ABO quartile to $77 \%(68-85 \%)$ in the highest $\left(P=0.006, \chi^{2}\right)$. Subgroup analysis showed that arrival ABO predicted patient access block, and that patient access block was associated with delay to surgery and longer postoperative length of stay (geometric mean 12.9 vs 9.9 days, $P<0.01$, $t$-test).

Conclusions: The number of access block patients at the time of arrival directly predicts delay to operation in this setting. This suggests that access block occupancy is a marker of hospital dysfunction.

Key words: $\quad$ Emergency, overcrowding, access block, fracture, delay.

Correspondence: 


\section{Introduction}

ED overcrowding is a proven risk factor for reduced ED function, ${ }^{1,2}$ decreased quality indicators ${ }^{3,4}$ and adverse patient outcomes. ${ }^{5,6}$ The predominant cause of overcrowding in Australasia is access block, ${ }^{7,8}$ the inability of ED patients requiring inpatient admission to access appropriate beds in a timely fashion. An excess of patients in the ED impairs the ability to assess and treat new patients, but it has been suggested that high ED occupancy, particularly with access block patients, reflects a whole-of-hospital dysfunction. ${ }^{6}$

The nationally accepted measure of access block, the proportion of patients admitted, transferred or died with a total $\mathrm{ED}$ time of more than $8 \mathrm{~h},{ }^{9}$ is by necessity calculated retrospectively over a period of time, usually a minimum of $24 \mathrm{~h}$ (presentation patterns vary during the day). Recent literature has suggested that the average occupancy with access block patients ('total access block hours') might be a better predictor of ED function. ${ }^{10}$ Neither of these measures clearly represent the state of the ED at any one point in time, and both might include the contribution of patients newly arrived during the period.

The present study sought to assess the point occupancy of an ED with access block patients as a measure of hospital dysfunction, using the care provided to a well-defined group of patients as a quality marker. The primary aim was to identify any relationship between the number of access block patients in the ED at the start of each hour and the time to definitive care of patients presenting with fractured neck of femur during that hour. Secondary aims were to investigate the relationship with other recognized quality of care measures. Fractured neck of femur is a high-risk condition, and an association is reported between delayed surgery and poor outcomes, ${ }^{11,12}$ but the relationship is complicated by preexisting illness. Nevertheless, many authorities recommend surgery within $24 \mathrm{~h}$ of hospital presentation. ${ }^{13,14}$

\section{Methods}

This was a retrospective descriptive cohort study carried out using chart review and existing data sources at The Canberra Hospital (TCH), Garran, Australian Capital Territory, a tertiary teaching hospital with a mixed ED seeing 52000 presentations annually. All presentations with a recorded ED diagnosis of fractured neck of femur in calendar years 2006 and 2007 were eligible for chart review. The unit of analysis was the presentation: it was assumed that separate fractures in separate hips were independent events if they occurred at different times in the same individual. Exclusion criteria on review were: age less than 50 years, misdiagnosis, no surgery and documented medical reason for surgical delay. All chart data were abstracted by one author (KM) blinded to the state of the ED, who recorded the exclusion criteria and the date and time of the definitive surgery from the theatre nurses' report and the anaesthetic sheet.

TCH ED keeps a long-term database of the number of ward inpatient access block patients in the $\mathrm{ED}$ (i.e. those being treated who have been in ED more than $8 \mathrm{~h}$ and are subsequently admitted to TCH beds, not including discharges from ED, deaths and transfers) derived from the hospital information system. These patients account for the vast majority of access block in this institution. This existing resource was used to derive the ED access block occupancy $(\mathrm{ABO}$, or number of ward inpatient access block patients in the ED) at the start of each hour for the 2 year period.

Second, quartiles for $\mathrm{ABO}$ at the start of each hour of the $24 \mathrm{~h}$ day were calculated from the 2 year dataset. Subsequently, each $1 \mathrm{~h}$ period of the 2 years was classified by quartile according to $\mathrm{ABO}$ at the start of the hour. These data were then merged with the abstracted time of surgery and patient/presentation data from the information system for eligible cases.

The time from ED arrival to start of surgery was calculated and classified as 'Timely' if less than $24 \mathrm{~h}$, and 'Delayed' if more than $24 \mathrm{~h}$. The primary hypothesis was that the proportion of delayed surgery would be greater in presentations occurring in higher $\mathrm{ABO}$ quartiles. Secondary analysis considered time to start of medical assessment and treatment in the ED, whether the individual patients experienced access block and postoperative length of stay (LOS) in hospital (calculated as a geometric mean because of skew).

Categorical data were compared with the $\chi^{2}$ test and continuous variables with the $t$-test, using a threshold of 0.05 for statistical significance. Power calculation showed that a sample size of 366 presentations would have $80 \%$ power to detect a difference between $50 \%$ and $65 \%$ delayed surgery between the lower two quartiles and the upper two quartiles.

\section{Results}

There were 442 presentations (by 437 different patients) with a recorded ED diagnosis of fractured neck of femur 
Table 1. Characteristics of sample

\begin{tabular}{lccr}
\hline & Number & Mean age (years) $(95 \% \mathrm{CI})$ & Male sex $(n, \%)$ \\
\hline Included & 369 & $80.1(79.0-81.2)$ & $109(29.5)$ \\
Age $<50$ years & 16 & $33.7(27.1-40.3)$ & $12(75)$ \\
Misdiagnosis & 5 & $77.6(70.4-84.8)$ & $0(0)$ \\
No surgery & 31 & $78.0(73.7-82.3)$ & $12(38.7)$ \\
Medical delay & 21 & $83.0(79.1-86.9)$ & $3(14.3)$ \\
Total & 442 & $78.4(77.1-79.7)$ & $136(30.7)$ \\
\hline
\end{tabular}

Baseline characteristics of the presentations for which chart review was undertaken. Only the characteristics of the group excluded for age $<50$ years were statistically significantly different from the population as a whole. 'Misdiagnosis' means that the recorded ED diagnosis of fractured neck of femur was incorrect, so neck of femur surgery was not performed.

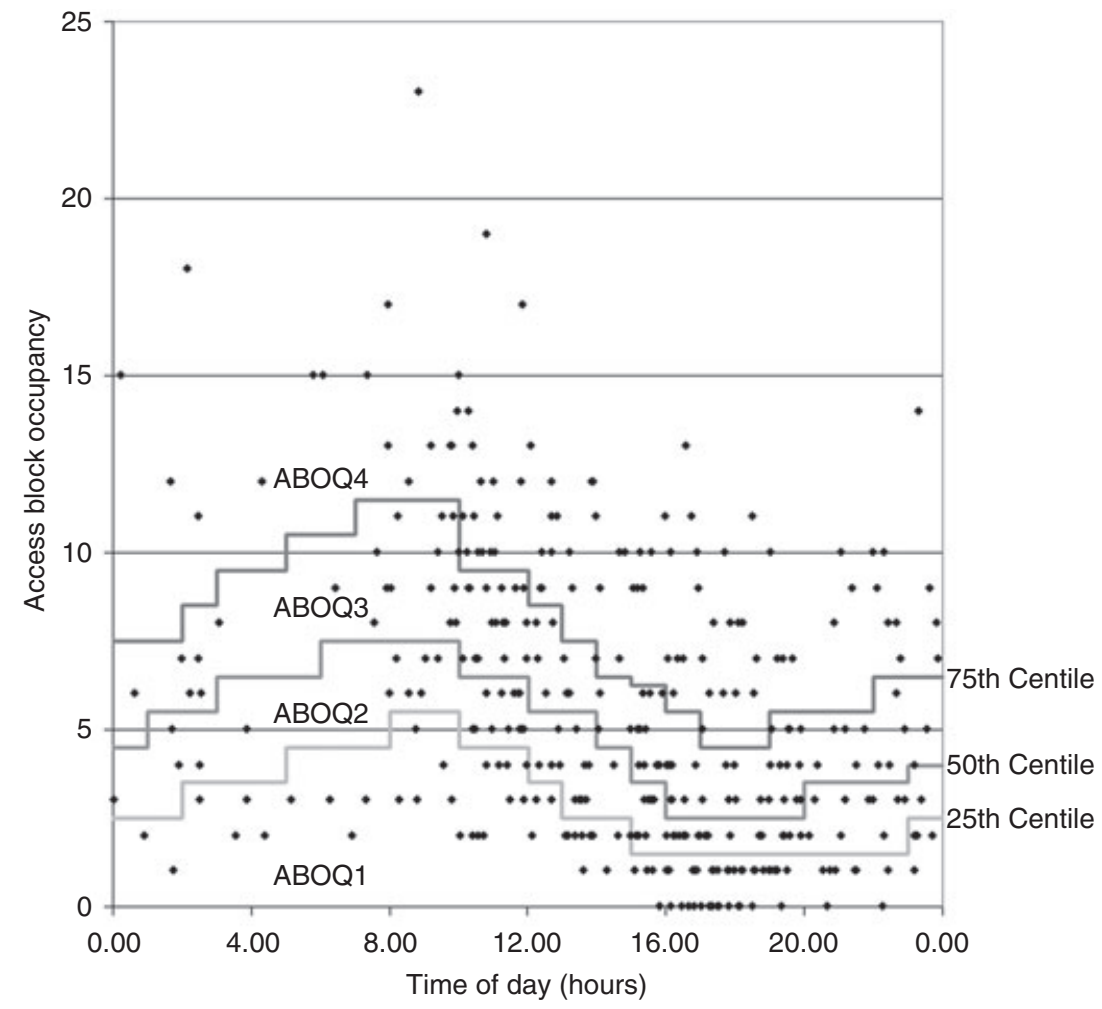

Figure 1. Time of presentation and access block occupancy (ABO) quartiles (Q). ABO quartiles (lines) for the 2 year period and time of presentation and actual $\mathrm{ABO}$ for the 369 cases in the study. The cases were evenly distributed among the quartiles.

in 2 years, with characteristics and exclusions as detailed in Table 1 . One hundred per cent of the 369 included charts were reviewed, and no significant change in the average rate of delayed surgery was found over the period of the study .Their times of presentation were evenly distributed among the $\mathrm{ABO}$ quartiles (Fig. 1), and there were no significant differences between the four groups demographically (Table 2). Triage (Australasian Triage Scale) was tested because a higher triage category implies an intent of shorter waiting time, and presentation times before 16.00 hours were tested because earlier arrival is associated with surgery on the same day. However, the differences in presentation time were not significant $\left(P=0.09, \chi^{2}\right)$, and the direction, if anything, would make presentations in ABO Q1 less likely to have timely surgery.

There was a significant association between $\mathrm{ABO}$ quartile at presentation and delayed surgery as shown 
Table 2. Characteristics by access block occupancy (ABO) quartiles

\begin{tabular}{lccccc}
\hline ABO quartile & Number & $\begin{array}{c}\text { Mean age (years) } \\
(95 \% \mathrm{CI})\end{array}$ & $\begin{array}{c}\text { Male sex } \\
(n, \%)\end{array}$ & $\begin{array}{c}\text { Australasian Triage Scale } \\
\text { category 3 }(n, \%)\end{array}$ & $\begin{array}{c}\text { Present }<16.00 \text { hours } \\
(n, \%)\end{array}$ \\
\hline 1 & 95 & $80.0(77.7-82.2)$ & $23(24)$ & $79(83)$ & $42(44)$ \\
2 & 79 & $80.0(77.4-82.6)$ & $26(33)$ & $62(78)$ & $45(57)$ \\
3 & 94 & $81.0(78.9-83.1)$ & $30(32)$ & $89(84)$ & $57(61)$ \\
4 & 101 & $79.7(77.6-81.6)$ & $30(30)$ & $83(82)$ & $60(59)$ \\
\hline
\end{tabular}

Characteristics of the four groups presenting in different $A B O$ quartiles.

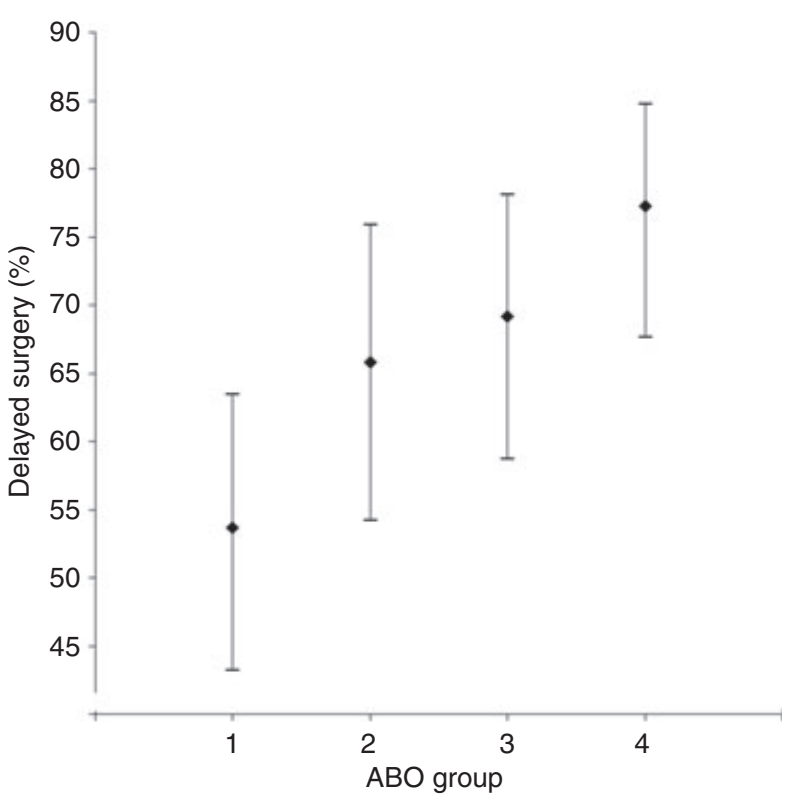

Figure 2. Delayed surgery. Proportion $( \pm 95 \% \mathrm{CI})$ of presentations resulting in delayed surgery ( $>24 \mathrm{~h}$ from arrival) by access block occupancy (ABO) group (quartile) at the time of presentation (note expanded scale).

in Figure $2\left(P=0.006, \chi^{2}\right)$. The relationship between ABO quartile and other markers of quality are shown in Table 3. There was a significant relationship between $\mathrm{ABO}$ quartile on arrival and the probability of the patient subsequently experiencing access block $(P<$ 0.001). There was no significant relationship between ABO quartile and subsequent postoperative LOS, but postoperative LOS was significantly higher in patients who experienced access block: geometric mean 12.9 versus 9.9 days, $P=0.009$, $t$-test.

\section{Discussion}

In this population, there is a significant increase in the incidence of delayed surgery with increasing ED ABO immediately before presentation. This is not due to the time of day nor to other recognized confounders, such as patient age and comorbidities.

The primary analysis of time from arrival to surgery does not distinguish between the function or dysfunction of different parts of the hospital system. The secondary analysis shows that $\mathrm{ABO}$ quartile on arrival does not predict ED waiting time, although waiting times for fractured neck of femur patients might not reflect ED function overall, as they are a highly select group $(100 \%$ of presentations in the present study arrived by ambulance). ABO quartile on arrival does predict whether the individual patient will experience access block, so it might be an indication of the state of the inpatient wards rather than the state of the ED.

The present study is consistent with previous work describing a relationship between individual access block and increased LOS, ${ }^{15,16}$ but was not designed to indicate the mechanism of this effect. The distribution of LOS is highly skewed, and confounded by the short LOS associated with mortality, so a larger and more detailed study would be required to identify the causes.

The subgroup analysis of other quality indicators suggests that the overall relationship between $\mathrm{ABO}$ quartile and hospital function is far from linear. Time spent in the ED ('patient care time') increases most dramatically between the third and fourth quartiles, but the greatest increase in time from leaving the ED to operation is between the first and second quartiles. Indeed, the time from ED to surgery actually decreases at extreme levels of $\mathrm{ABO}$, perhaps because of external reactions, such as cancellation of elective cases, which would make the operating theatres more readily accessible. ABO quartiles represent a useful means of controlling for daily variation, but absolute occupancy figures might provide a more accurate reflection of the situation in ED.

The present study is limited by its retrospective methodology, but certainly provides justification for future prospective studies. The choice of a $24 \mathrm{~h}$ threshold from arrival to surgery was arbitrary, and the time 
Table 3. Process measures by access block occupancy (ABO) quartile

\begin{tabular}{lccccc}
\hline $\begin{array}{l}\text { ABO } \\
\text { quartile }\end{array}$ & $\begin{array}{c}\text { Access } \\
\text { block }(\%)\end{array}$ & $\begin{array}{c}\text { Median waiting } \\
\text { time }(\mathrm{h})\end{array}$ & $\begin{array}{c}\text { Median patient } \\
\text { care time }(\mathrm{h})\end{array}$ & $\begin{array}{c}\text { Median left ED } \\
\text { to surgery (h) }\end{array}$ & $\begin{array}{c}\text { Median postoperative } \\
\text { LOS (days) }\end{array}$ \\
\hline 1 & 46 & $0: 31(0: 14-1: 05)$ & $6: 39(4: 14-10: 19)$ & $17: 08(11: 44-35: 44)$ & $10(5-17.5)$ \\
2 & 49 & $0: 37(0: 19-1: 33)$ & $6: 34(3: 54-13: 21)$ & $21: 06(12: 13-40: 33)$ & $10(5-23.5)$ \\
3 & 57 & $0: 37(0: 11-1: 21)$ & $8: 19(4: 08-13: 41)$ & $21: 09(11: 37-41: 18)$ & $10(7-16)$ \\
4 & 77 & $0: 31(0: 14-1: 14)$ & $14: 46(7: 37-21: 34)$ & $18: 27(8: 00-36: 24)$ & $10(6-20)$ \\
All & 58 & $0: 34(0: 14-1: 15)$ & $8: 19(4: 42-15: 56)$ & $18: 42(10: 45-39: 22)$ & $10(6-19)$
\end{tabular}

Standard measures of patient flow ${ }^{9}$ by $A B O$ quartile on arrival; figures in brackets are interquartile ranges. Access block is the proportion of patients with total ED Time $>8 \mathrm{~h}$, waiting time is the time from arrival to start of assessment and treatment, patient care time from start of assessment/treatment until left ED and LOS is total nights in hospital after surgery (this was highly skewed).

from injury to surgery might represent a better indicator of quality of care, but the time of injury was infrequently recorded in the patient notes.

In terms of patient care, fractured neck of femur is not a condition that improves with conservative management: it is clearly in the interests of hospital function to start the postoperative care as soon as possible. The present study justifies a prospective trial of measures designed to reduce access block.

\section{Conclusions}

The occupancy of the ED with access block patients immediately before the arrival of a patient with fractured neck of femur directly predicts lesser hospital quality of care as defined by the incidence of delay to surgery. ABO might therefore be a useful measure or indicator of hospital dysfunction.

\section{Competing interests}

None declared.

Accepted 1 June 2009

\section{References}

1. Weiss SJ, Ernst AA, Nick TG. Relationship between the National ED overcrowding scale and the number of patients who leave without being seen in an academic ED. Am.J. Emerg. Med. 2005; 23: 288-94

2. Dunn R. Reduced access block causes shorter emergency department waiting times: an historical control observational study. Emerg. Med. (Fremantle) 2003; 15: 232-8.

3. Pines JM, Hollander JE, Localio AR et al. The association between emergency department crowding and hospital performance on antibiotic timing for pneumonia and percutaneous intervention for myocardial infarction. Acad. Emerg. Med. 2006; 13: 873-8.

4. Hwang U, Richardson LD, Sonuyi TO, Morrison RS. The effect of emergency department crowding on the management of pain in older adults with hip fracture. J. Am. Geriatr. Soc. 2006; 54: $270-5$.

5. Sprivulis PC, Da Silva JA, Jacobs IG et al. The association between hospital overcrowding and mortality among patients admitted via Western Australian emergency departments. Med. J. Aust. 2006; 184: 208-12.

6. Richardson DB. Increase in patient mortality at 10 days associated with emergency department overcrowding. Med. J. Aust. 2006; 184: 213-16.

7. Fatovich DM, Nagree Y, Sprivulis P. Access block causes emergency department overcrowding and ambulance diversion in Perth, Western Australia. Emerg. Med. J. 2005; 22: 351-4.

8. Cameron PA, Campbell DA. Access block: problems and progress. Med. J. Aust. 2003; 178: 99-109.

9. Australasian College for Emergency Medicine. Policy document - standard terminology. Emerg. Med. Australas. 2002; 14: 33740.

10. Paoloni R, Fowler D. Total access block time: a comprehensive and intuitive way to measure the total effect of access block on the emergency department. Emerg. Med. Australas. 2008; 20: $16-22$.

11. Siegmeth AW, Gurusamy K, Parker MJ. Delay to surgery prolongs hospital stay in patients with fractures of the proximal femur. J. Bone Joint Surg. Br. 2005; 87: 1123-6.

12. Bottle A, Aylin P. Mortality associated with delay in operation after hip fracture: observational study. BMJ 2006; 332: 947-51.

13. Turner P, Cocks J, Cade R, Ewing H, Collopy B, Thompson G. Fractured neck of the femur (DRG 210/211): prospective outcome study. Aust. N. Z. J. Surg. 1997; 67: 126-30.

14. Elliott J, Beringer T, Kee F, Marsh D, Willis C, Stevenson M. Predicting survival after treatment for fracture of the proximal femur and the effect of delays to surgery. J. Clin. Epidemiol. 2003; 56: 788-95.

15. Liew D, Liew D, Kennedy MP. Emergency department length of stay independently predicts excess inpatient length of stay. Med. J. Aust. 2003; 179: 524-6.

16. Richardson DB. The access-block effect: relationship between delay to reaching an inpatient bed and inpatient length of stay. Med. J. Aust. 2002; 177: 492-5. 